Pezzeta, S. Ciencias sociales y derecho: las ciencias sociales en la educación legal. Derecho y Ciencias Sociales. Abril 2018. N ${ }^{o}$ 18. Pgs 183-198 ISNN 1852-2971. Instituto de Cultura Jurídica y Maestría en Sociología Jurídica. FCJ y S. UNLP

\title{
Ciencias sociales y derecho: las ciencias sociales en la educación legal
}

\author{
Social sciences and law: social sciences in legal education
}

\section{Silvina Pezzeta}

\section{Resumen}

En este trabajo presentaré a manera de hipótesis algunos de los problemas que surgen en las relaciones entre el derecho, como disciplina del conocimiento, y las ciencias sociales. A tal fin haré una aproximación lingüística a la cuestión siguiendo, para ello, los trabajos de Elizabeth Mertz sobre la educación legal en los Estados Unidos y sus reflexiones teóricas sobre el rol que tienen en ésta las ciencias sociales. El trabajo se dividirá en dos secciones. En la primera, presentaré algunas ideas centrales sobre cuestiones referidas a los estudios del lenguaje en uso y sobre los problemas de "traducción" de las ciencias sociales para emplear sus hallazgos y teorías en el campo del derecho. Asimismo, presentaré los resultados de la mencionada autora respecto de la enseñanza del derecho y rol que ocupan las ciencias sociales en ésta. En la segunda parte me abocaré a consideraciones sobre la perspectiva de la dogmática y la diferencia que supone respecto de las ciencias sociales para, finalmente, explorar los desafíos de desarrollar una enseñanza del derecho que no dependa exclusivamente de la práctica pero que, a la vez, sea relevante para ella. Se trata de presentar argumentos para explorar en el futuro un modelo de educación jurídica en la que las ciencias sociales no ocupen un lugar ornamental ni sean mero instrumento de los fines derivados de la práctica.

\section{Palabras claves}

Ciencias sociales; Educación legal; Dogmática

\begin{abstract}
In this work I will offer an approach to some of the problems that arise in the relations between social science and jurisprudence. In order to achieve the goals, I will use a linguistic approach following Elizabeth Mertz research about legal education and her reflections about the role that social sciences have on it. The work will be developed in two sections. In the first one, there will be presented some crucial ideas about studies of language use and the translations problems that appear when scholars try to apply social sciences results and theories in the legal realm. Then, I will present the results of Mertz's investigation about legal education and how social sciences are taught in American law schools. In the second section I will make some critical considerations about jurisprudence and will show some differences when compared with social sciences to, finally, explore the challenges that has to face a legal education that intends to be both independent from practice and, at the same time, relevant to it. I will show, then, some reasons to try to develop a legal education model in which social sciences are not a mere ornament nor a something strictly derive from practice itself.
\end{abstract}

\section{Key Words}

Social Sciences; Legal Education; Jurisprudence

\footnotetext{
- Doctora en Derecho, Investigadora Asistente CONICET, profesora Facultad de Derecho, UBA. silvinapezzetta@gmail.com
} 
Pezzeta, S. Ciencias sociales y derecho: las ciencias sociales en la educación legal. Derecho y Ciencias Sociales. Abril 2018. N ${ }^{\circ}$ 18. Pgs 183-198 ISNN 1852-2971. Instituto de Cultura Jurídica y Maestría en Sociología Jurídica. FCJ y S. UNLP

\section{Ciencias sociales y derecho: las ciencias sociales en la educación legal}

\section{Silvina Pezzeta}

\section{Introducción}

En este trabajo abordaré el problema de las relaciones entre el derecho, como disciplina del conocimiento, y las ciencias sociales. A tal fin haré una aproximación lingüística a la cuestión siguiendo, para ello, los trabajos de Elizabeth Mertz sobre la educación legal en los Estados Unidos y sus reflexiones teóricas sobre el rol que tienen en ésta las ciencias sociales. El trabajo se dividirá en dos secciones. En la primera presentaré algunas ideas centrales sobre cuestiones referidas a los estudios del lenguaje en uso y sobre los problemas de traducción de las ciencias sociales en el campo del derecho. Asimismo, presentaré los resultados de la mencionada autora respecto de la enseñanza del derecho. En estos se puede ver que los profesores y estudiantes emplean distintos marcos interpretativos del lenguaje según se refieran al derecho o a cuestiones tratadas por las ciencias sociales. En la segunda parte me abocaré a consideraciones sobre la perspectiva de la dogmática y la diferencia que supone respecto de las ciencias sociales para, finalmente, explorar los desafíos de desarrollar una enseñanza del derecho que no dependa exclusivamente de la práctica pero que, a la vez, sea relevante para ella. La propuesta será brindar algunos argumentos a fin de lograr una educación jurídica en la que las ciencias sociales no ocupen ni un lugar ornamental ni sean mero instrumento de los fines derivados de la práctica (Lista y Brígido, 2012; Kennedy, 1995; Lista y Begala, 2003; Begala y Lista 2014; Gordon, 2004; Manzo, 2008).

\section{Ciencias sociales y derecho: tres problemas}

Se pueden distinguir al menos tres problemas cuando se reflexiona acerca de las relaciones entre el derecho -como disciplina del conocimiento ligada fuertemente a una práctica social de carácter autoritativo- y las ciencias sociales ${ }^{1}$. Los tres tienen un origen común: la necesidad del derecho de recurrir a otras disciplinas para dar respuestas a sus

\footnotetext{
${ }^{1}$ Estos tres problemas que aquí esbozo son el producto de la experiencia personal como parte del campo y también de mi trabajo de investigación sobre la enseñanza del derecho y, a su vez, se apoyan en un determinado criterio normativo sobre qué deberíamos enseñar y cómo deberíamos investigar. Aquí, los tres problemas funcionan como motores de inspiración en la propuesta de utilizar los estudios del lenguaje en uso para aproximarnos aspectos como la educación legal, las decisiones judiciales y la investigación jurídica, aunque sin pretender que éstos sean los únicos posibles.
} 
Pezzeta, S. Ciencias sociales y derecho: las ciencias sociales en la educación legal. Derecho y Ciencias Sociales. Abril 2018. N ${ }^{\circ}$ 18. Pgs 183-198 ISNN 1852-2971. Instituto de Cultura Jurídica y Maestría en Sociología Jurídica. FCJ y S. UNLP

desafíos y objetivos. El primer problema es el de las relaciones entre las ciencias sociales, que tienen una cantidad de requisitos epistémicos y metodológicos específicos, y la forma más tradicional de producir conocimiento jurídico, la dogmática o doctrina ${ }^{2}$, que se sostiene en base a una lógica diferente. Esta primera cuestión adquiere especial visibilidad cuando los abogados se proponen abordar proyectos de investigación, o desarrollar tesis doctorales, que involucran objetivos empíricos. Las dificultades se observan, especialmente, en la construcción de marcos teóricos y en la explicitación de la metodología, pasos exigidos en todos los formularios de planes de trabajo. Estos requisitos se contraponen a lo que se aprendió a través de las tradicionales lecturas que socializan a los estudiantes de derecho y abogados. En general, la bibliografía consiste en manuales y comentarios de doctrinarios que trabajan con un estilo que no incluye un armado teórico explícito. El segundo problema es el de los usos de los resultados de las ciencias sociales, pero también de las naturales o exactas, en la práctica del derecho: ¿cómo se interpreta la indeterminación de la ciencia social? ¿Y la incertidumbre de las ciencias naturales? ¿Qué sucede con los distintos paradigmas de las ciencias sociales en el ámbito jurídico? ¿Cómo se establecen los criterios de confiabilidad en materia científica? Por último, el tercer problema es qué lugar ocupan, y qué lugar deberían ocupar, las ciencias sociales en el curriculum universitario de derecho.

Los tres problemas, si bien distintos, están conectados y pueden ser abordados desde una perspectiva que preste atención al lenguaje. En particular, aquí me abocaré al primer y segundo problema y lo haré siguiendo la perspectiva teórica y los trabajos de Elizabeth Mertz (2007; 2008; 2011a; 2011b) antropóloga lingüística y abogada. Sus investigaciones utilizan un marco teórico compuesto de conceptos utilizados para investigar el lenguaje en uso y la forma en que distintos marcos interpretativos influyen en la comprensión de los estilos y fines diferentes que orientan a las ciencias sociales y a la ciencia del derecho, y cómo esto repercute en la enseñanza universitaria. La aproximación elegida encuentra su fundamento en la importancia que tiene el uso del lenguaje en los distintos ámbitos de interacción social en los que contribuye a generar el sentido. En efecto, un sinnúmero de actividades que llevamos adelante en nuestra vida diaria no serían posibles sin el uso del lenguaje. Éste es central en los casos más distintos, desde el mantenimiento de las relaciones sociales a la expresión de nuestros sentimientos, la comunicación de ideas sobre el mundo y, además, lo es también para generar cambios en la realidad como cuando se emiten determinadas fórmulas específicas que tienen carácter performativo. Incluso, para muchos autores, nuestra experiencia está mediada

\footnotetext{
${ }^{2}$ Aquí no me refiero a la filosofía del derecho, porque no es objeto de este trabajo, que tiene sus propias exigencias epistémicas y teóricas y que, por supuesto, difiere de la dogmática tradicional.
} 
Pezzeta, S. Ciencias sociales y derecho: las ciencias sociales en la educación legal. Derecho y Ciencias Sociales. Abril 2018. N ${ }^{\circ}$ 18. Pgs 183-198 ISNN 1852-2971. Instituto de Cultura Jurídica y Maestría en Sociología Jurídica. FCJ y S. UNLP

por el lenguaje de forma tal que la determina en gran medida. Durante los años 60, como señalan Angermuller, Mainguenau y Wodak (2014), emergió un nuevo campo de investigación, todavía en plena actividad, que gira en torno al discurso y que se preocupa del lenguaje no en sus aspectos descontextualizados sino en su uso real y situado (Lavandera, 1985). Dos grandes teorías del lenguaje influyeron en este campo, el estructuralismo y la pragmática. En ambos casos se trata de maneras distintas de estudiar la producción social del sentido. Para el estructuralismo lo crucial respecto del sentido es el conjunto de reglas gramaticales; para el pragmatismo, el significado es el producto de la actividad lingüística llevada adelante en un contexto determinado (Angermuller, Mainguenau y Wodak, 2014: 17).

Cada una de estas corrientes inspiró distintas teorías e investigaciones que se enfocaron en aspectos tales como las reglas de la conversación, la cortesía, las implicaturas conversacionales, el carácter indexical del lenguaje, la multifuncionalidad del lenguaje, las ideologías lingüísticas, la variación lingüística y la polifonía, entre muchos otros. Estos temas constituyeron, a su vez, los objetos de diferentes disciplinas como el análisis del discurso, la antropología lingüística, la sociolingüística, la etnografía del habla, la pragmática y la teoría de la enunciación, por ejemplo. En todos los casos se trata de prestar atención al lenguaje utilizado y no, al menos no exclusivamente, a los aspectos contextualmente independientes del lenguaje (Calsamiglia Blancáfort y Tusón Valls, 2002). Aquí estoy interesada en dos conceptos que utiliza Mertz: el nivel metalingüístico y la ideología del lenguaje. Ambos permitirán entender algunos aspectos de las difíciles relaciones entre la ciencia dogmática tradicional y otros tipos de conocimiento, como el que producen las ciencias sociales. En el siguiente apartado me referiré a ellos proveyendo, además, un brevísimo marco conceptual que permita ubicarlos en la discusión sobre la relevancia del lenguaje para entender estas conflictivas relaciones.

\subsection{Lenguaje y metalenguaje: marco interpretativo e ideología lingüística}

En esta sección voy a seguir específicamente el trabajo de Mertz, "La subestimación de la indeterminación: traducciones de la ciencia social para el campo jurídico" (Mertz, 2011) en el que compara los distintos marcos interpretativos que orientan a abogados y científicos sociales. La autora se propone reflexionar sobre el problema de la comunicación entre ellos en lo específicamente referido a la interpretación de los alcances de los resultados de las investigaciones sociales. En particular, le interesa explicar por qué a los abogados les resulta 
Pezzeta, S. Ciencias sociales y derecho: las ciencias sociales en la educación legal. Derecho y Ciencias Sociales. Abril 2018. N ${ }^{\circ}$ 18. Pgs 183-198 ISNN 1852-2971. Instituto de Cultura Jurídica y Maestría en Sociología Jurídica. FCJ y S. UNLP

difícil no estimar negativamente la indeterminación propia de los resultados de las ciencias sociales. Presenta el problema como uno de traducción y comunicación, que es una forma típica de pensar problemas en la antropología. Para ella, la cuestión es de especial relevancia porque los inconvenientes de comunicación son importantes en la utilización de la ciencia social en casos de familia o de la aplicación de la pena de muerte, por ejemplo. En estas circunstancias aparecen problemas en la interpretación que hacen los actores judiciales de los resultados de las investigaciones científicas. Las interpretaciones se realizan apoyadas en marcos interpretativos que operan en el nivel metalingüístico y están asociados, además, a ideologías lingüísticas.

Las teorías que se ocupan del lenguaje han prestado una especial atención al nivel metalingüístico. La diferencia entre lenguaje objeto y metalenguaje permitieron avances en lógica y filosofía analítica. Pero aquí interesa especialmente la teoría de Roman Jakobson (1984) sobre la multifuncionalidad del lenguaje porque ésta fue de especial importancia para los estudios del lenguaje en uso. Jakobson (1984) describió seis funciones: descriptiva, conativa, fática, expresiva, poética y metalingüística. El lenguaje, entonces, tiene la capacidad de llevar a cabo diferentes funciones según con qué elemento constituyente esté involucrado: emisor, receptor, canal, código, referente o mensaje. El nivel metalingüístico, en el que opera el marco interpretativo, debe ser comprendido en el marco de esta teoría. Es importante hacer notar que de estas seis funciones la función referencial, o descriptiva, es la que parece más rápidamente accesible a la conciencia lingüística. Esta función, que consiste en la propiedad del lenguaje de referirse al contexto extralingüístico, ha sido considerada la principal del lenguaje por muchos filósofos. Sin embargo, las demás funciones tienen tanto peso como ésta. La función expresiva surge de la orientación del lenguaje hacia el propio enunciador y sirve para manifestar opiniones y emociones. Si el lenguaje se dirige al intérprete, se cumple la función conativa como, por ejemplo, cuando se hace una pregunta o se da una orden. A veces el lenguaje se dirige a sí mismo para resaltar sonidos y regularidades, como cuando se rima. Esta es la función poética según Jakobson. Ésta abarca todos aquellos usos del lenguaje en que se resaltan sus propias características estéticas. Si la relación del lenguaje está dirigida al canal a través del que es posible la comunicación, la función se denomina fática o de contacto. El objetivo de la función fática es mantener la comunicación, asegurarse de que el canal está funcionando correctamente. La función metalingüística tiene lugar cuando el lenguaje se dirige a sí mismo, cuando se toma a sí mismo por objeto. Esta función puede subdividirse en dos clases: metasemántica y metapragmática. En el primer caso se trata de la referencia al contenido semántico de alguna porción del lenguaje (como cuando aclaramos qué significa 
Pezzeta, S. Ciencias sociales y derecho: las ciencias sociales en la educación legal. Derecho y Ciencias Sociales. Abril 2018. N ${ }^{\circ}$ 18. Pgs 183-198 ISNN 1852-2971. Instituto de Cultura Jurídica y Maestría en Sociología Jurídica. FCJ y S. UNLP

una palabra). En el segundo, la función es metapragmática porque se refiere a cuestiones de uso en contexto (como cuando se afirma que no es correcto tratar de "vos" a una persona mayor).

El segundo aspecto central en el estudio del lenguaje en uso es el de la ideología lingüísticas y está estrechamente relacionado con la función metalingüística (Lucy, 1993: 9). De hecho, se trata de conjuntos de ideas no neutrales sobre el lenguaje que pueden referirse tanto a éste como un todo, a algunos aspectos estructurales del mismo o a sus usos y funciones. Se trata, también, de un fenómeno metalingüístico puesto que toma al lenguaje como objeto. Una ideología lingüística prevaleciente es la que sostiene que la función central del lenguaje, o la única incluso, es la referencial. Austin denominó a esta suposición sobre la finalidad predominante del lenguaje "falacia descriptiva". El nivel metalingüístico, y la ideología lingüística, son determinantes en el lenguaje porque exceden en mucho la mera referencia al lenguaje objeto. En el nivel metalingüístico se establecen las pistas o claves para que una participación sea interpretada de una manera determinada: como broma, narración, insulto o explicación, por ejemplo. El nivel metalingüístico es el que guía la forma en que se interpretan los discursos y Mertz indica que en este se constituyen los diversos marcos interpretativos.

La función metalingüística sobrepasa, entonces, lo señalado por Jakobson (1984), quien la reducía a una mera referencia al código (que además consideraba común al receptor y al emisor). Se trata, antes bien de la consagración, e incluso la imposición en algunos casos, de un tipo de "código" determinado. Por lo tanto, no sólo tiene una función referencial sino también performativa. Analizar lo que sucede en este nivel permite acceder entonces a las relaciones entre lenguaje y poder. Así lo señalan, por ejemplo, Mertz y Yovel (2010:4) cuando se refieren a las relaciones entre lenguaje objeto o código y el nivel metalingüístico: “QQué hablantes e instituciones están en condiciones de enmarcar, formar y dictar el "código" -el vocabulario, la gramática, la poética y otros aspectos de la interacción lingüística que se suponen independientes del evento comunicativo-? ¿Qué características estructurales e ideológicas tanto tácitas como manifiestas moldean el discurso y cómo?"

En suma, y desde una perspectiva preocupada por el lenguaje en uso, el nivel metalingüístico es un aspecto central en cualquier investigación o reflexión teórica para echar luz sobre problemas como el de la comunicación entre miembros de dos grupos que usan el lenguaje de manera muy distinta. En el siguiente apartado me referiré a los resultados de la investigación que llevó a cabo Mertz sobre la educación legal en los Estados Unidos. Entre estos se destaca el hallazgo sobre cómo los profesores de derecho operan con un marco 
Pezzeta, S. Ciencias sociales y derecho: las ciencias sociales en la educación legal. Derecho y Ciencias Sociales. Abril 2018. N ${ }^{\circ}$ 18. Pgs 183-198 ISNN 1852-2971. Instituto de Cultura Jurídica y Maestría en Sociología Jurídica. FCJ y S. UNLP

interpretativo que establece diferencias en la estructura lingüística cuando se refieren al derecho o a los problemas sociales y las ciencias sociales. De esta manera, se produce un interesante efecto que puede cristalizarse en una ideología lingüística respecto de las funciones del lenguaje en el ámbito jurídico que, en la segunda sección de este trabajo, trasladaré al ámbito local pero en cuanto a las relaciones entre dogmática y ciencias sociales y sólo como pretensión de presentar argumentos que deberán ser explorados en futuros desarrollos.

\subsection{Problemas legales y contexto social en la educación jurídica en Estados Unidos}

En su libro "El lenguaje de la facultad de Derecho. Aprender a pensar como un abogado" (Mertz, 2007), la autora presenta los resultados de una investigación de campo sobre la forma en que se enseña derecho en los Estados Unidos. Gracias a su formación en antropología lingüística y en derecho, fue capaz de plantear de manera original una pregunta clásica en el ámbito de la teoría del derecho: qué es el derecho. Es interesante notar que señala respecto del punto de vista antropológico que éste no parte de la idea de que existe una respuesta única a una pregunta de esta clase, ni sus objetivos consisten en buscar algo semejante (Mertz, 2007: 13). Más aún, es muy importante considerar el punto de vista de los propios participantes de la práctica, en este caso, de la práctica jurídica. El título de la obra da cuenta de esta aproximación al utilizar una frase propia del mundo de los abogados, "pensar como un abogado", que resume lo que sería una característica definitoria que comparten de manera uniforme quienes estudiaron derecho. Las preguntas que orientaron la investigación, precisamente, buscaron establecer lo que Mertz denomina "la epistemología jurídica" que opera en las aulas de derecho, tanto en sus semejanzas como en sus diferencias, así como la estructura lingüística de la pedagogía jurídica. Por último, otro objetivo, que aquí no desarrollaré, fue dilucidar las diferencias entre los distintos grupos seleccionados en función de una variedad de factores que operan como condicionantes -como la raza o el género- de mayor o menor participación de los estudiantes.

Una de las características salientes del trabajo es la extensión del objeto recortado, que incluyó ocho facultades de derecho, seleccionadas de acuerdo a un criterio de jerarquía propio de los mismos participantes. De esta forma, entre las escuelas había tres consideradas de élite, dos con estatus regional y tres con prestigio meramente local. Se optó por hacer observación no participante de cursos de una misma materia dictada en primer año, Contratos, para evitar 
Pezzeta, S. Ciencias sociales y derecho: las ciencias sociales en la educación legal. Derecho y Ciencias Sociales. Abril 2018. N ${ }^{\circ}$ 18. Pgs 183-198 ISNN 1852-2971. Instituto de Cultura Jurídica y Maestría en Sociología Jurídica. FCJ y S. UNLP

que las disparidades que se encontraran obedecieran al tipo de disciplina enseñada en lugar de otros factores relevantes que sí estaban siendo revisados (como el género o la raza de los profesores, por ejemplo). Un equipo conformado por la autora y estudiantes de sociología o antropología, especialmente entrenados al efecto, grabó las clases y tomó notas durante las mismas durante todo el semestre. A este material se sumó el producido en pequeños grupos focales y entrevistas con seis profesores. Por último, también se utilizaron datos secundarios cuantitativos para dotar de mayor contexto a los datos etnográficos.

El marco teórico de la investigación estuvo compuesto por una serie de teorías que buscan elaborar herramientas que permitan estudiar el lenguaje en uso, es decir, el lenguaje no en sus aspectos descontextualizados o meramente semánticos, sino las estructuras, regularidades y fenómenos propios de las interacciones verbales reales. Para ponerlo en términos históricos, es prestar atención a lo que Saussure (1945) denominó "habla" -en contraposición a la "lengua" como sistema de signos-. En esta línea, son muy importantes los detalles precisos de las interacciones verbales y es a partir de ellos que, de manera inductiva, se obtiene una descripción de las regularidades, y diferencias, observables en el "lenguaje del derecho" con el que se socializa a los estudiantes de primer año. Mertz explica que la enseñanza de una nueva forma de pensar se logra a través de una nueva manera de leer, escribir y hablar. Esta forma supone, entonces, una nueva manera de seleccionar detalles y referirse a las historias y dramas humanos que aparecen en un nuevo paisaje, el legal.

Según muestra en las conclusiones Mertz (2007: 207), lo común a todas las escuelas de derecho es una epistemología o cosmovisión jurídica que enseña esta nueva forma de leer, hablar y escribir como abogados a través de un mensaje que es acerca de la estructura pragmática o contextual y que se transmite a partir de las características pragmáticas del lenguaje usado en las clases. Esto demuestra que no sólo es importante la cuestión semántica de lo que se transmite, sino que los detalles contextuales, o pragmáticos, de la interacción son centrales en tanto que guían la nueva interpretación requerida para abordar los textos. Se observa aquí la importancia del nivel metalingüístico (meta pragmático, en este caso) que queda de manifiesto a través de la observación no participante. Mertz se concentró en varios aspectos pragmáticos y meta pragmáticos, entre los cuáles se encuentra la estructura de "uptakes"- que aquí se traducirá como "cita directa" aunque no es exactamente lo mismo, sino que es una admisión en el discurso propio de la respuesta del participante de la interacción cuando ésta coincide con lo esperado-. Esta admisión, o su falta, tienen en distintos contextos diferentes sentidos. En el caso de las facultades de derecho, la estructura pragmática del lenguaje en uso es el diálogo (real o ficticio del propio profesor que introduce respuestas de 
Pezzeta, S. Ciencias sociales y derecho: las ciencias sociales en la educación legal. Derecho y Ciencias Sociales. Abril 2018. N ${ }^{\circ}$ 18. Pgs 183-198 ISNN 1852-2971. Instituto de Cultura Jurídica y Maestría en Sociología Jurídica. FCJ y S. UNLP

otras voces en su discurso) en el que hay cita directa sólo cuando una estudiante produce la respuesta correcta. Así, los profesores ignoran todos los detalles sociales, dramáticos o emotivos de las respuestas de sus alumnos y sólo admiten en su discurso lo que los estudiantes dicen, con estricta precisión, sobre qué tribunal dijo qué cosa. Esta forma pragmática es mucho más sutil para introducir un nuevo marco interpretativo con el cual aproximarse a los textos que una estrategia que, por ejemplo, corrigiera explícitamente a los estudiantes cuando responden equivocadamente las preguntas de los profesores.

Mertz (2007: 8) explica en estos términos la cosmovisión o epistemología que se transmite en las clases a través de la estructura pragmática descubierta: "Cuando los estudiantes intentan contar las historias de los conflictos de los casos que les fueron asignados empiezan, típicamente, contando el contenido de la historia en cuestión. Los profesores de primer año, insistentemente, los redireccionan hacia la autoridad que entendió en ese caso. ¿Cuál fue el tribunal autorizado para decidir sobre ese caso?”

Se les exige a los estudiantes un altísimo nivel de exactitud para referirse, además, a estos nuevos aspectos de las historias que, al inicio del semestre de las clases observadas, no eran los que espontáneamente reproducían en sus respuestas. Los profesores sólo toman las palabras de sus estudiantes cuando repiten exactamente lo que quieren que aprendan: qué autoridad dijo qué cosa, qué instancia fue, cómo se relaciona con el precedente. Mertz señala que, en contraste, durante las clases también se podía abrir un espacio para la discusión de cuestiones como el contexto social -una vez que hubiera terminado el intenso y puntual debate sobre cuestiones estrictamente legales-. En estas interacciones entre estudiantes y profesores sobre problemas sociales, de discriminación racial o de género, por ejemplo, se permitían todo tipo de especulaciones y estaba ausente la precisión en la cita de investigaciones o autores. El intercambio lingüístico era relajado y fluido, los detalles sociales sólo eran importantes si eran útiles para las categorías legales relevantes según el marco interpretativo utilizado en los intercambios legales sobre los casos asignados.

En suma, la forma en que se enseña derecho descansa en los detalles pragmáticos y meta pragmáticos de la interacción entre profesores y estudiantes en el aula. Aquí me interesa lo común a todas las clases observadas que consiste en la enseñanza de una nueva manera de leer y hablar que focaliza en aspectos específicos de los casos que deben preparar los estudiantes para las clases. Mertz (2007: 20) señala respecto del lenguaje de las aulas de derecho lo siguiente: “...el foco en la forma del lenguaje crea un sistema lingüístico muy cerrado que es capaz, en esencia, de engullir todos los detalles sociales sin que eso lo afecte. Por contraste, al menos algunos tipos de ciencias sociales demandan a los investigadores que 
Pezzeta, S. Ciencias sociales y derecho: las ciencias sociales en la educación legal. Derecho y Ciencias Sociales. Abril 2018. N ${ }^{\circ}$ 18. Pgs 183-198 ISNN 1852-2971. Instituto de Cultura Jurídica y Maestría en Sociología Jurídica. FCJ y S. UNLP

estén abiertos a revisar sus supuestos más firmes. Si los datos entran en conflicto con su teoría, desafortunadamente, en el largo plazo, es probable que la teoría, y no los datos, sea lo que será dejado de lado. Por el contrario, un abogado debe apoyar los intereses de su cliente y desafiar cualquier dato que encuentre que no lo favorezca".

En el siguiente apartado presentaré algunos detalles de la dogmática tradicional utilizando este tipo de aproximación teórica para compararla, en función de sus marcos interpretativos y del concepto de ideología lingüística, con las ciencias sociales.

\section{Sobre la dogmática jurídica y las ciencias sociales: de objetivos y marcos teóricos}

La dogmática jurídica, doctrina o ciencia del derecho, tiene una larga tradición que antecede incluso a la aparición de la ciencia moderna. Durante siglos se erigió como una forma legítima de conocimiento, siendo inclusive una de las disciplinas sobre las que se desarrollaron las universidades. Sin embargo, a partir de los éxitos de la biología y la física en la Edad Moderna, la ciencia jurídica debió tomar posición respecto de estas formas de conocimiento, con las que tenía poco en común. Para ello, los teóricos del derecho se apoyaron en distintas estrategias, desde una comparación que los posicionaba como científicos, hasta la afirmación de que se trataba de dos tipos de conocimientos diferentes, cada uno con sus propios criterios de validación. En la actualidad, frente a un desarrollo de las ciencias sociales y la filosofía que presenta un paisaje de líneas divisorias borrosas entre las tareas de explicación/comprensión/descripción y las de evaluación y análisis conceptual, la actividad de los juristas puede ser pensada con más armonía respecto del resto de las disciplinas científico-sociales. No obstante, a pesar de los cambios, la distancia entre ambas tradiciones persiste y se pueden observar, por ejemplo, en las dificultades de los alumnos de postgrado, especialmente de doctorado, para desarrollar proyectos de investigación que requieren de la utilización de un marco teórico explícitamente estipulado.

Es muy relevante para este trabajo señalar uno de los motivos por los que las ciencias sociales adquieren típicamente un lugar instrumental u ornamental en los planes de estudio de abogacía: la falta de comprensión sobre las diferencias entre la forma de construir conocimientos de las ciencias sociales y la de la dogmática. (Carrió, 1957; Begala, 2014; Nino, 2014). Propongo aquí utilizar la idea de Mertz sobre que el origen de estas dificultades, al menos parcialmente, en los diferentes marcos lingüísticos que cada una de ellas utilizan.

La discusión sobre el carácter disciplinar y epistemológico de los estudios sobre el derecho ha atravesado distintas etapas que han sido bien caracterizadas en los textos de 
Pezzeta, S. Ciencias sociales y derecho: las ciencias sociales en la educación legal. Derecho y Ciencias Sociales. Abril 2018. N ${ }^{\circ}$ 18. Pgs 183-198 ISNN 1852-2971. Instituto de Cultura Jurídica y Maestría en Sociología Jurídica. FCJ y S. UNLP

historia de la filosofía del derecho (Pattaro, 1980), por ejemplo, así como también en las propuestas normativas de distintos autores sobre qué tipo de investigación es el más conveniente. A pesar de que la discusión sobre el estatus epistemológico de la llamada ciencia jurídica no es en estos momentos de especial interés, como tampoco lo es el debate sobre la definición del derecho que suele estar asociada a ella, se trata de un tema clásico que está en la base de muchos de los problemas jurídicos que obtienen más atención en la actualidad. En este apartado sólo relevaré algunas características generales de este tipo estudios del derecho. Esto servirá para dar un contexto descriptivo a los temas que he revisado en los apartados anteriores que se ofrecen a manera de hipótesis explicativa de las conflictivas relaciones entre el derecho y las ciencias sociales: que los distintos marcos lingüísticos e ideologías lingüísticas implicadas en uno y otras son uno de los obstáculos al momento de generar buenas relaciones entre ambos. Antes de dar algunas de las características de la dogmática jurídica tradicional es necesario aclarar que existen otras clases de trabajos jurídicos que son más afines a los lineamientos y parámetros epistemológicos de las ciencias sociales o de la filosofía, según sea el caso. Incluso más, muchos trabajos que cumplen la misma función de la dogmática se realizan, no obstante, en consonancia con algunos de los parámetros de la investigación. Sin embargo, el peso de la dogmática tradicional es aún muy importante en la enseñanza del derecho que todavía se apoya, en gran parte, en textos de este tipo.

La dogmática, o ciencia del derecho, es una actividad que Carlos Nino (2014) describió en varios de sus trabajos caracterizándola como el producto de una mezcla entre dos intenciones contradictorias que detentarían quienes trabajan en esta línea. Por un lado, los dogmáticos adhieren al derecho positivo sólo por ser tal, sin cuestionar su conveniencia, justicia, coherencia, oportunidad u otros aspectos. Esto los haría pasibles de ser considerados positivistas ideológicos, posición que él rechazaba. Pero, por otra parte, los dogmáticos también ofrecen soluciones a los problemas habituales que se encuentran a la hora de interpretar y aplicar el derecho tales como la indeterminación del lenguaje jurídico y los problemas de incoherencia entre normas, entre otros. Para ello, muchas veces van más allá de señalar los problemas para ofrecer soluciones que esconden, por ejemplo, criterios normativos. La finalidad de los dogmáticos es escribir para hacer algo más que meramente reproducir lo que el derecho positivo dice, algo que, por lo demás, sería escasamente útil. El objetivo de los dogmáticos es, igual que su objeto de estudio, resolver conflictos. En este caso, resolver conflictos interpretativos para los que ofrecen soluciones. Uno de los mayores problemas para Nino es que hagan esto último pretendiendo derivar esta opinión del sistema jurídico sin más, como si la solución estuviera allí incluida de manera explícita y clara. Esto 
Pezzeta, S. Ciencias sociales y derecho: las ciencias sociales en la educación legal. Derecho y Ciencias Sociales. Abril 2018. N ${ }^{\circ}$ 18. Pgs 183-198 ISNN 1852-2971. Instituto de Cultura Jurídica y Maestría en Sociología Jurídica. FCJ y S. UNLP

suele hacerse a partir de una serie de recursos bien conocidos como la atribución al legislador de características como la racionalidad, la omnisciencia, la coherencia y otras necesarias para lograr la solución que parezca más adecuada.

Esta caracterización, que considero útil si se la toma como un tipo ideal, permite entender en qué difiere esta manera de producir conocimiento jurídico de las producciones de conocimiento científico sociales. En este sentido, lo que hay que destacar es el marco interpretativo, o nivel metalingüístico, de la dogmática: la interpretación de las normas o del sistema normativo se hace para ofrecer soluciones para la aplicación del derecho. Debido a que el interés es eminentemente práctico, la reflexividad, la especulación y la espera de más avances en la investigación para llegar a una solución o conclusión, no son posibles ni deseables. Se puede trazar entonces en este punto la diferencia con las ciencias sociales, que están orientadas por un interés principal en producir conocimiento confiable. Por ello, la construcción del marco teórico es el paso más importante en ciencias sociales. Y el marco teórico, que no es otra cosa que un nivel meta lingüístico, es objeto de reflexión consciente en éstas. En contraposición, los abogados suelen trabajar también en un nivel metalingüístico, pero sobre el que existe menos reflexividad. El objetivo de los trabajos dogmáticos tradicionales es práctico: ofrecer soluciones o criticar algunas dadas por legisladores o jueces. Estos distintos marcos interpretativos operan como ideologías lingüísticas en tanto son invisibles y generan problemas para la comprensión de otros tipos de conocimientos que, en lo particular, interfieren en la elaboración de los planes de estudio de derecho. Sobre ello tratará el siguiente apartado.

\section{A manera de cierre: breves ideas sobre la integración de las ciencias sociales en el currículum de derecho. Ni ornamento ni mera herramienta}

Para algunos autores (Bovino y Courtis, 2009: 303) no cabe pensar en otro modelo de estudios sobre el derecho que no sean los dogmáticos debido a que suponen que la diferencia entre tipos de conocimiento está basada en el objeto ${ }^{3}$. Alegan que el derecho está dirigido a ordenar comportamientos sociales y que, por ello, la dogmática tiene obviamente una finalidad práctica, como si existiera un nexo lógico entre uno y otra. Acá sostengo que esa orientación de la dogmática es sólo una posibilidad, asentada sobre una ideología linguiística

\footnotetext{
${ }^{3}$ Esto es lo que sostienen Bovino y Courtis aunque no creo que exista un nexo lógico entre el objeto de estudio y el tipo de estudios posibles sobre esto. Afirmar tal cosa sería como decir que la investigación sobre la religión convierte en religiosos a sus autores. De hecho, el objeto de estudio jurídico merece ser estudiado desde distintos puntos de vistas y disciplinas, algunos de ellos buscarán una finalidad práctica como la dogmática mientras que otros tendrán sobre este objeto un interés puramente teórico.
} 
Pezzeta, S. Ciencias sociales y derecho: las ciencias sociales en la educación legal. Derecho y Ciencias Sociales. Abril 2018. N ${ }^{\circ}$ 18. Pgs 183-198 ISNN 1852-2971. Instituto de Cultura Jurídica y Maestría en Sociología Jurídica. FCJ y S. UNLP

del uso del lenguaje en el ámbito legal. Es decir, el marco interpretativo que orienta a los abogados en nuestro caso opera de manera tal que aparece como un obstáculo para la comprensión de otros usos del lenguaje, como el de las ciencias sociales. A esto hay que agregar un aspecto que suele hacer más complicada la comunicación: la creencia extendida entre muchos abogados respecto del estatus científico que tendrían también los estudios dogmáticos. Esta creencia es a veces compartida por los mismos dogmáticos quienes, a la vez, llegan a veces a reconocer que se trata de trabajos que se orientan a resolver problemas prácticos en los que, además, juegan un papel importante los juicios valorativos (explícitos o encubiertos).

Me interesa señalar que los distintos marcos lingüísticos y los diferentes objetivos entre los tipos de conocimientos que comparé constituyen un buen punto de partida para pensar en los obstáculos para el desarrollo de otra clase de planes de estudio para las facultades de derecho. Idealmente, estos planes de estudio deberían incorporar asignaturas propias de otras disciplinas sin que esto se haga bajo la lógica del mero ornamento o de una simple provisión de instrumentos para los futuros abogados litigantes o funcionarios judiciales. De hecho, dada la fuerte influencia que tiene la dogmática sobre la forma de estudiar el derecho que se considera legítima -una que tenga en cuenta la práctica litigiosa y la resolución judicial de los conflictos normados- las asignaturas "no codificadas" y "no jurídicas" suelen oscilar entre esos extremos. En este sentido, quizás sea la filosofía del derecho la que más ha avanzado en articular trabajos con un fuerte marco teórico y una agenda de temas propios. Al hacerlo, claro, se aleja de muchas preocupaciones cotidianas de los que participan en el desenvolvimiento diario del derecho en instancias como el litigio. Sin embargo, gracias a la posibilidad de tomar distancia y plantear con rigurosidad los problemas a partir de un marco teórico bien constituido, puede proyectar nuevas formas de comprensión y, en última instancia, también nuevas soluciones jurídicas.

Para pensar en cómo enseñar derecho sin estos sesgos tradicionales que limitan el campo de interés de los abogados un camino posible es recurrir al campo de producción del conocimiento jurídico, donde aparecen muy buenos y prometedores trabajos por fuera de esta lógica. Las facultades de derecho, como recontextualizadoras del conocimiento, podrían tomar estas nuevas formas para incorporar asignaturas y seminarios que provean de una nueva formación jurídica. En ese orden de cosas creo necesario hacer algunas referencias sobre este tipo de estudios que adoptan una serie de pautas que los coloca más cerca de las ciencias sociales. Estos trabajos, que muchos se apresuran a catalogar de sociología jurídica, tienen la particularidad de utilizar un marco teórico o analítico que toma elementos que van más allá de 
Pezzeta, S. Ciencias sociales y derecho: las ciencias sociales en la educación legal. Derecho y Ciencias Sociales. Abril 2018. N ${ }^{\circ}$ 18. Pgs 183-198 ISNN 1852-2971. Instituto de Cultura Jurídica y Maestría en Sociología Jurídica. FCJ y S. UNLP

las teorías del derecho tradicionales. Para ello, acuden sin ortodoxia a la teoría social, la sociología, la filosofía, por ejemplo, para construir el objeto derecho de una manera que excede el tradicional concepto formalista de la dogmática jurídica. Una vez reconstruido el objeto, aparecen nuevos interrogantes y objetivos que no están orientados directamente a resolver los problemas que aparecen en la práctica cotidiana sino aquellos que los mismos investigadores conciben y que son problemas teóricos o empíricos que buscan producir conocimiento. Estos estudios suelen hacerse a partir de la reflexión sobre dos aspectos: el carácter de los estudios tradicionales dogmáticos y sus límites y la necesidad de acudir a otras disciplinas para investigar en derecho.

Quiero finalizar este trabajo argumentando a favor de un mayor desarrollo de este tipo de investigaciones en una línea muy similar a la que sostienen César Rodríguez Garavito y Mauricio Villegas (2003), autores que hace ya varios años promueven el desarrollo de estudios jurídicos interdisciplinarios y críticos. Para ellos, que destacan el desarrollo de una creciente cantidad de esta clase de estudios en América Latina, la tarea necesaria es romper con los esquemas disciplinarios impuestos por la ciencia moderna y el formalismo. Esto está en consonancia con las nuevas características del panorama de la teoría social actual (Siedman y Alexander, 2004), en la que las tareas descriptivas y explicativas no dejan de lado objetivos más propios de la filosofía práctica o del activismo, por ejemplo. Rodríguez Garavito y García Villegas (2003)proponen que estos trabajos deben adquirir mayor importancia en América Latina y para ello es necesario establecer redes que conecten a sus autores, así como una agenda de temas y una epistemología crítica, además de tener como objetivo la promoción de la emancipación y una sociedad igualitaria.

Agregaría que también deben tener impacto en los diseños curriculares. Quiero notar aquí que los aspectos normativos y prescriptivos serían más fácilmente comprensibles para el marco lingüístico propio de los abogados. Sin embargo, es preciso acentuar que los trabajos que señalan los autores sí requieren del manejo de fuertes instrumentos teóricos tales como un marco analítico y una metodología específica. Los autores señalan tres temas de investigación que deberían abordarse en nuestra región por representar rasgos definitorios de la misma: la pluralidad jurídica, la ineficacia del derecho y el autoritarismo. Pero, además, también incluyen otros tópicos estudiados que rebalsan los límites habituales de la ciencia jurídica tradicional que son el género, los pueblos originarios, las reformas constitucionales, los derechos humanos y la globalización, enumeración a la que se podría sumar el problema de la igualdad, la violencia institucional y los derechos de los animales, por ejemplo. Para todos ellos, como parece claro, será inevitable acudir a disciplinas extra dogmáticas y, entonces, 
Pezzeta, S. Ciencias sociales y derecho: las ciencias sociales en la educación legal. Derecho y Ciencias Sociales. Abril 2018. N ${ }^{\circ}$ 18. Pgs 183-198 ISNN 1852-2971. Instituto de Cultura Jurídica y Maestría en Sociología Jurídica. FCJ y S. UNLP

será necesario comprender los distintos marcos metalingüísticos que orientan la construcción de conocimiento a los efectos de realizar las traducciones de manera adecuada. Y evitar, en suma, un rol de las ciencias sociales limitado al ornamento o el instrumental.

\section{Bibliografía}

Angermuller, J, Mainguenau, D y Wodak, R. (2014). The discourse reader. Main currents in theory and analysis., Amsterdam-Philadelphia: John Benjamins Publishing Company.

Austin, J. L. (1962). Cómo hacer cosas con palabras. Barcelona: Paidós.

Begala, S y Lista C.. (2014). La sociología como campo de conocimiento especializado en la formación de abogados. En: https://www.academia.edu/33291797/La_sociolog\%C3\%ADa_jur\%C3\%ADdica_como_camp o_de_conocimiento_especializado_en_la_formaci\%C3\%B3n_de_los_abogados.pdf

Begala, S. (2003). "La presencia del mensaje educativo en la conciencia de los estudiantes: resultados de la socialización en un modelo jurídico dominante", en Revista Academia, Año 1, Número 2 .

Bovino, A. y Courtis, Christian. (2009). "Por una dogmática conscientemente política", en Desde otra mirada. Textos de teoría crítica del Derecho. Buenos Aires: Eudeba, 2da. Edición.

Calsamiglia Blancáfort, H y Tusón Valls, A. (2002). Las cosas del decir. Manual de análisis del discurso, Barcelona: Ed. Ariel.

Carrió, G. (1957). "Sobre las creencias de los juristas y la ciencia del derecho", en Revista Lecciones y Ensayos, Vol. VI, pp. 27-37.

García Villegas, M. y Rodríguez Garavito, C. (2003). "Derecho y sociedad en América Latina: propuestas para la consolidación de los estudios jurídicos críticos", en Derecho y sociedad en América Latina. Un debate sobre los estudios jurídicos críticos.

Jakobson, R. (1984), Ensayos de lingüística general, Barcelona, Ariel.

Kennedy, D. (1995). Politizicing the classroom. En: http://www.duncankennedy.net/documents/Politicizing\%20the\%20Classroom.pdf

Lavandera, B. (1985). Curso de lingüística para el análisis del discurso, Buenos Aires: Centro Editor para América Latina.

Lista., C. y Begala, S. (2005). "El discurso jurídico en la ensenianza jurídica: entre lo instruccional y lo regulativo", en VI Congreso de Sociología Jurídica, Buenos Aires,

Lista, C. y Brígido, C. (2002). La enseñanza del derecho y la formación de la conciencia jurídica, Córdoba: Sima Ediciones.

Lucy, J. (1993). "Reflexive language and the human disciplines", en Reflexive language. Reported speech and metapragmatics. New York: Cambridge University Press.

Manzo, M. (2008). "La influencia de la educación jurídica en la formación valorativa de los abogados", en Revista Academia, Año 6, Nro. 11

Mertz, E. (2007). "Inside the law school classroom: toward a new legal realist pedagogy". En: http://ssrn.com/abstract $=1719235$

Mertz, E. (2010). The language of the law school. Learning to think like a lawyer. La Verge (USA): Oxford University Press. 
Pezzeta, S. Ciencias sociales y derecho: las ciencias sociales en la educación legal. Derecho y Ciencias Sociales. Abril 2018. N ${ }^{\circ}$ 18. Pgs 183-198 ISNN 1852-2971. Instituto de Cultura Jurídica y Maestría en Sociología Jurídica. FCJ y S. UNLP

Mertz, E. (2008). "The role of social science in law: introduction". En: htpp://ssrn.com/abstract=1711222

Mertz, E. (2011a). "Social science and the intellectual apprenticeship: moving the scholarly mission of law school forward”. En: http://ssrn.com/abstract=1992532

Mertz, E. (2011b). "Undervaluing indeterminacy: translating social science into law". En: http://ssrn.com/abstract=1988179

Mertz, E. y Yovel, J. "Metalinguistic awareness", en The handbook of pragmatics, Ed. JanOla Ostman, Jef Verschueren, Jan Blommaert, Chris Bulcaen. Disponible en: http://works.bepress.com/jonathan_yovel/15/.P.4.

Nino, C.S. (2014) Introducción al análisis del Derecho. Buenos Aires, 2da. Edición ampliada y revisada, 17ava. Reimpresión: Astrea.

Pattaro, E. (1980). Filosofía del derecho. Derecho y ciencia jurídica. Madrid: Reus.

Siedman, S. y Alexander, J. (2004). The new social theory reader. Contemporary debates, New York, Routledge. 